\title{
Energy Optimized Parameterization of Spherical Triangle Mesh
}

\author{
Chao Sun, Zhenbo Guo \\ College of Information Engineering \\ Qingdao University \\ Qingdao, China \\ sunchao6106@163.com/gzb@qdu.edu.cn
}

\author{
Kaixi Wang, Na Cheng \\ College of Information Engineering \\ Qingdao University \\ Qingdao, China \\ kxwang@qdu.edu.cn/cn17863908021@163.com
}

\begin{abstract}
Parametric deformation is the reference to measure the quality of the parameters. According to it, a threedimensional mesh spherical parameterzation method is put forward based on local deformation energy for arbitrary and spherical homeomorphism of zero defect lattice close type grid model, and the parameter domain is extended from planar region to spherical region. Experimental results show that the algorithm is simple, fast and accurate.
\end{abstract}

Keywords-Spherical Parameterization; Distortion of Energy; Triangular Mesh.

\section{INTRODUCTION}

With the continuous development of modern computer technology, 3D scanners, aerospace simulation technology, medical identification, engineering construction and other applications to promote. Triangular meshes with its efficiency, simplicity and other characteristics, has become the mainstream method of complex three-dimensional model of expression [1].

Triangular mesh model has been complicated by the threedimensional surface through the sampling and re-establishing of the vertex 3D scanners.

Problem is equivalent to [2, 3]: Given a triangular mesh surface $\mathrm{S}$ by the $3 \mathrm{D}$ point set consisting $v_{i} \in R^{3}$ and a twodimensional flow parameter domain D. Looking for a point $u_{i} \in D$ to $v_{i} \in S$ bijection $\psi$ on the parameter field, so that the mesh and the original mesh topology parameter domains are isomorphic, and ensuring that all maps do not overlap the triangular piece on the parameter field. In this situation, then searching some inner parameter domain mapping triangle between the sheet and the original mesh geometry to minimize distortion measure. Common geometric metrics include edge length, angle, and area. Bijection guarantees the uniqueness from the three-dimensional mesh surface to the two-dimensional parameter field and from the two-dimensional parameter field to the three-dimensional mesh surface mapping, which simplifies the complexity of the operation at the same time.

According to the perspective of examining the problem, the triangular mesh parameterization methods basically have the following classifications:

- Depending on the deformation of the internal geometry metric, divided into area parameterization; isometric and conformal parameterization.
- Depending on the complexity of computing time to solve, divided into non-linear and linear.

- $\quad$ Depending on the mapping parameter field, divided into flat and spherical parameterization.

\section{PREVIOUS WORKS}

The research of the method of planar parameter domain is divided into two categories: one kind of grid boundary has been defined and is convex polygon, and the other is a closed grid or in any forms.

For the closed mesh topology, the way of taking a plane parameterization process, parameterized will undoubtedly become very complex, requires fragmentation cutting process, and the cutting line will lead to the original discrete data gird topology, produce a large deformation. Intuitively mapped mesh data to texture mapping plane domain is the most direct and most natural way, but for the mesh topology is equivalent to a sphere, the parameters of the embryo to its topological sphere is more reasonable and effective. The connection between the three-dimensional mesh topology mapping onto the spherical region, ensures no overlapping spherical polygon.

In recent years, the parameterization of the sphere has become a hot research topic in the research of the triangular mesh parameterization. Kent [4] proposed a simple spherical parameterization. By the expansion of the balloon, the triangular mesh is properly attached to the sphere. The experimental results can satisfy the purpose of optimization, but it is lack of basic theory; Alex [5] proposed a relaxation parametric method based on the theory of elasticity. In this method, all points on the grid are mapped to the minimal enclosing sphere of the model, and then the coordinates of the 6 vertices of the sphere are maintained, and the other vertices of the spherical surface are smoothed by the discrete Laplace averaging operator; Kobbelt [6] proposed a similar partial smoothing method of relaxation. However, these methods have a common defect, lack of theoretical argument, assumption is very strong, and no consideration angle deformation; Shapiro [7] proposed the idea of a simplified grid-based rapid convergence to complete the mapping. The method by deleting the 1-ring center which order less than 6, re-triangulation cavity formed by the delete operation, until the original for the tetrahedral mesh simplification. Eventually tetrahedron simplified mapping to a sphere, and added the deleted vertices on the spherical topology by the inverse operation. The method can fast convergence, faster, able to quickly handle complex 
grid, but the points which selected to delete, affect the entire quality of texture mapping.

\section{APPROACH OVERVIEW}

Parametric can be seen a bijection between the surface and the parameter field plane. Triangular mesh with its advantages, has now become one of the mainstream model complex 3D surface representation. For a parameter mapping, as the rear triangle mesh topology is isomorphic to the original triangle mesh topology, which is called effective parametric. Details as follows:

- $\quad$ Triangular mesh topology on the original surface and triangular mesh mapped by parameter fields after the parameter mapping is bijective.

- Parameter domain mapping the original surface triangles do not overlap with each other. As shown in Figure 1, Q is a valid point of parametric mapping for the original $3 \mathrm{D}$ triangular mesh model, but $Q^{\prime}$ is invalid.

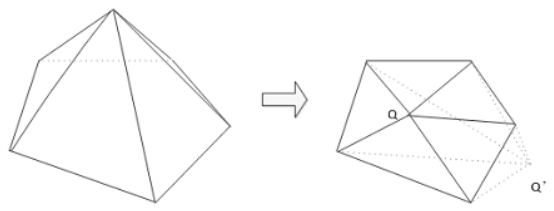

Fig. 1 The effectiveness of triangular mesh parameterization.

The quality of parameter mapping method is generally deformed by the parameter deformation size to measure. Intuitive visual through human eye is the most direct qualitative criteria of the parameter mapping deformation measurement. Here are two kinds of quantitative criteria to measure the deformation parameterization mapping:

- A parametric deformation is measured by the relative deviation of the area and the angle between the mesh topology of the spherical domain and the mesh topology of the original 3D surface. Deviation measurement [8]:

$$
\begin{aligned}
& \text { Diff } f_{\text {area }}^{2}(P)=\sqrt{\sum_{j}\left[\frac{\operatorname{Area}\left(T_{j}\right)}{\sum_{T_{j} \in M} \operatorname{Area}\left(T_{j}\right)}-\frac{\operatorname{Area}\left(T_{j}^{*}\right)}{\sum_{T_{j}^{*} \in P} \operatorname{Area}\left(T_{j}^{*}\right)}\right]^{2}} \\
& \operatorname{Diff}_{\text {angle }}^{2}(P)=\sqrt{\sum_{j}\left[\sum_{i=1}^{3}\left[\frac{\operatorname{Angle}\left(A_{i, j}\right)}{2 \pi}-\frac{\operatorname{Angle}\left(A_{i, j}^{*}\right)}{2 \pi+e}\right]^{2}\right]}
\end{aligned}
$$

Where $\mathrm{j}$ is a triangle mesh subscript; $\mathrm{M}$ is the set of the original triangle mesh, $\operatorname{Area}\left(T_{j}\right)$ for the $\mathrm{j}$-th original triangle area, and $\operatorname{Area}\left(T_{j}^{*}\right)$ for the $\mathrm{j}$-th parameterization triangle region area; Angle $\left(A_{i, j}\right)$ for the $\mathrm{i}$-th angle of the $\mathrm{j}$-th original triangle, and $\operatorname{Angle}\left(A_{i, j}^{*}\right)$ for the i-th angle of the $\mathrm{j}$-th parameterization domain triangle; e represents the surplus of spherical triangle.

- $\quad$ The maximum relative deviation of angle and area between the mesh topology of the spherical and the original $3 \mathrm{D}$ surfaces are used to measure the deformation [2]:

$$
\operatorname{Diff} f_{\text {area }}^{\infty}(P)=\max _{j}\left[\left|\frac{\operatorname{Area}\left(T_{j}\right)}{\sum_{T_{j} \in M} \operatorname{Area}\left(T_{j}\right)}-\frac{\operatorname{Area}\left(T_{j}^{*}\right)}{\sum_{T_{j}^{*} \in P} A \operatorname{Area}\left(T_{j}^{*}\right)}\right|\right]
$$

$$
\operatorname{Diff} f_{\text {angle }}^{\infty}(\mathrm{P})=\max _{\mathrm{j}}\left(\sum_{i=1}^{3}\left[\frac{\operatorname{Angle}\left(A_{i, j}\right)}{2 \pi}-\frac{\operatorname{Angle}\left(A_{i, j}^{*}\right)}{2 \pi+e}\right]^{2}\right)
$$

\section{LOCAL PARAMETERIZED ALGORITHM}

Parametric process constitute:

- $\quad$ Performing iterative edge collapse operation until the current mesh is a convex polyhedron. Vertices which each edge collapse operation to delete are recorded to the progressive grid.

- $\quad$ Obtaining an intermediate spherical parameterization grid which obtained by the simplified convex polyhedron are mapped to the unit ball. According to the progressive mesh detail records and the deformation energy of local parameterization, performing the vertex split operations, the generated two new vertices onto the unit sphere.

A. Quicken progressive meshes

In this paper, using Quicken [9] edge collapse simplification algorithm to generate a progressive mesh PM. Algorithm obtains the convex polygons by a continuous side folding method. When performing side folding operation, deleting the two vertices edges, as well as two vertices are parameterized mapped to the spherical triangle grid, and the mapping details are recorded in the progressive mesh. Detail records ensures that a simplified mesh $M^{0}$ can be a step by step to return to the initial mesh $M^{n}$. The reaction process vertex split operation. Every time division operation, it adds a vertex to the grid, the recording resume progressive mesh vertex position is based on details of the above records.
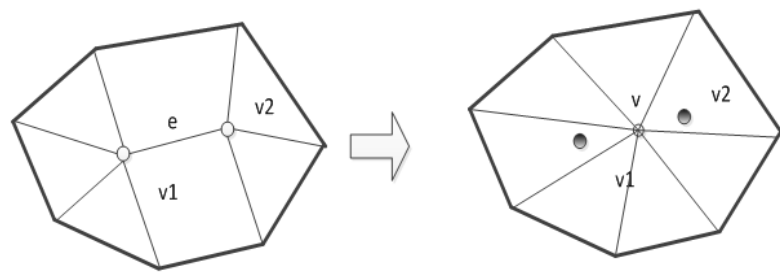

Fig. 2 The operation of spherical edge collapse.

\section{B. Local deformation energy}

The geometric deformation energy which this article proposed evolves from flat to spherical domain field, which is used for local deformation optimization. If it is not only a conformal mapping but also insurance area, it is the best parameter mapping. However, there is always the reality on the deformation of angle or area, and geometric parameters of the essence is to make the smallest measure deformation. Deformation energy metric of plane domain as follows [10]:

$$
\begin{aligned}
& \mathrm{E}(\mathrm{T})=\varepsilon \cdot E_{\text {angle }}(T)+(1-\varepsilon) \cdot E_{\text {area }}(T), \quad \varepsilon \in[0,1] \\
& f(q)=\frac{\operatorname{Area}\left(\Delta q q_{2} q_{3}\right) p_{1}+\operatorname{Area}\left(\Delta q_{1} q q_{2}\right) p_{2}+\operatorname{Area}\left(\Delta q_{1} q_{2} q\right) p_{3}}{\operatorname{Area}\left(\Delta q_{1} q_{2} q_{3}\right)} \\
& E_{\text {angle }}(T)=\left|\frac{\partial f}{\partial u}+i \frac{\partial f}{\partial v}\right|^{2} \\
& E_{\text {area }}(T)=|\operatorname{det} I-1|^{2}
\end{aligned}
$$




$$
\begin{gathered}
\frac{\partial f}{\partial \mathrm{v}}=\frac{\left(u_{3}-u_{2}\right)\left(x_{1}+i y_{1}\right)+\left(u_{1}-u_{3}\right)\left(x_{2}+i y_{2}\right)+\left(u_{2}-u_{1}\right)\left(x_{3}+i y_{3}\right)}{2 \operatorname{Area}\left(\Delta q_{1} q_{2} q_{3}\right)} \\
\frac{\partial f}{\partial \mathrm{u}}=\frac{\left(v_{3}-v_{2}\right)\left(x_{1}+i y_{1}\right)+\left(v_{1}-v_{3}\right)\left(x_{2}+i y_{2}\right)+\left(v_{2}-v_{1}\right)\left(x_{3}+i y_{3}\right)}{2 \operatorname{Area}\left(\Delta q_{1} q_{2} q_{3}\right)}
\end{gathered}
$$

$\mathrm{f}$ is the plane affine parameter domain mapping relationship to the original $3 \mathrm{D}$ triangular mesh surface; $(u, v)$ is the coordinate of plane parameter fields; $\mathrm{I}=\left[\begin{array}{ll}\frac{\partial f}{\partial u} \cdot \frac{\partial f}{\partial u} & \frac{\partial f}{\partial u} \cdot \frac{\partial f}{\partial v} \\ \frac{\partial f}{\partial u} \cdot \frac{\partial f}{\partial v} & \frac{\partial f}{\partial v} \cdot \frac{\partial f}{\partial v}\end{array}\right] ;\left(p_{1}, p_{2}, p_{3}\right)$ are the three points of a triangle original piece of 3D surfaces, $\left(\mathrm{q}_{1}, \mathrm{q}_{2}, \mathrm{q}_{3}\right)$ corresponds to its three points in the domain mapping parameters of triangle; Area(*) corresponds to its area of triangle; $(\mathrm{x}, \mathrm{y})$ as a local orthogonal coordinate base point of the original surface. From the above formula, the process is too complicated to calculate, and the quantity is very large. Therefore, this article presents a simple, quick calculation of deformation energy metric:

$$
\begin{aligned}
& \mathrm{E}(\mathrm{T})=\varepsilon \mathrm{E}_{\text {len }}(\mathrm{T})+(1-\varepsilon) \mathrm{E}_{\text {area }}(\mathrm{T}), \quad \varepsilon \in[0,1] \\
& \mathrm{E}_{\text {len }}(\mathrm{T})=\sum \frac{\left(\mathrm{Qlen}\left(\mathrm{q}_{\mathrm{i}}-\mathrm{q}_{\mathrm{j}}\right)^{2}-\left\|\mathrm{p}_{\mathrm{i}}-\mathrm{p}_{\mathrm{j}}\right\|^{2}\right)^{2}}{\left\|\mathrm{p}_{\mathrm{i}}-\mathrm{p}_{\mathrm{j}}\right\|^{2}} \\
& \mathrm{E}_{\text {area }}(\mathrm{T})=\frac{\left.\sum_{\mathrm{j}}\left[\sum_{\mathrm{i}=1}^{3} \text { Angle }(\mathrm{j}, \mathrm{i})\right)-\pi\right]}{\mathrm{n} \pi}
\end{aligned}
$$

Where Qlen(*) corresponds the original surface points in the arc sphere; Angle $(j, i)$ corresponds the $j$-th triangle's $i$-th angle, as described below:

By spherical harmonic transform $\mathrm{Y}_{1}^{\mathrm{m}}(\theta, \varphi)$, the original 3D triangular surfaces are normalized to unit inside the ball, and with the basics of the spherical triangle, spherical ball on the unit area of the triangle $A B C$ 's area $=A+B+C-\pi$, wherein the angle $A$ is equivalent to the angle between the plane of point A, O, B and the plane where A, O, C lies. Similarly rest. The article takes $\varepsilon=\frac{1}{2}$, the arithmetic mean value of the angle deformation energy and the area deformation energy as the final part of the deformation energy measurement.

\section{Experimental results and analysis}

Figure 3 shows the results of the algorithm based on progressive mesh method for spherical parameterization mapping of Rabbit model. The figure shows that the eyes and mouth, and other Rabbit mesh feature region have carried out adequate sampling grid, but non-feature area density distribution is not obvious.

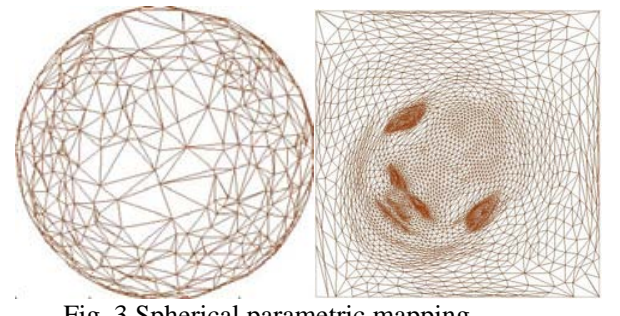

Fig. 3 Spherical parametric mapping.
Figure 4 shows the inverse transformation of the spherical triangle mesh (base mesh model). Seen from the figure, the base model of the inverse transform is better to maintain the characteristic part of the original model.

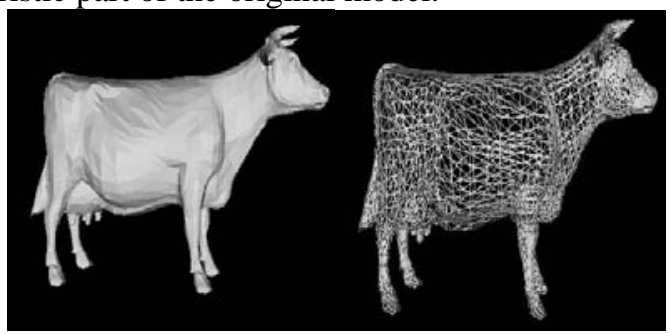

Fig. 4 The result of parametric inverse process.

The verification of this algorithm is still not perfect, and the overall guidance of the global deformation energy is short. In some cases, a step the local optimal solution is not necessarily equivalent to the final optimal solution, which need to be further improved in the future.

\section{SUMMARY}

The local deformation energy is extended to the sphere parameterization based on the planar parameterization domain, and the problem of the spherical parameterization of the closed triangular mesh is solved. While the solution process is fast. Simultaneously, there are also some shortcomings.

- $\quad$ The choice of local deformation energy parameter $\varepsilon$ is not to make a specific case study based on different grid.

- Global deformation energy has no effective verification as to whether the local optimal solution is equivalent to the global optimal solution.

In this paper, parametric deformation is the standard to measure the quality of the parameters. Based on this standard, for arbitrary and spherical homeomorphism of zero defect lattice close type grid model, a three-dimensional mesh spherical parameterization method based on local deformation energy is designed, the parameter domain extends from planar region to spherical region. Experimental results show that the algorithm is simple, fast and accurate.

This paper's purpose is simply getting a concise method when performing planar parametric operation. It is mainly focused on the topology of closed mesh. In this case, the spherical parameterization is the most appropriate and natural mapping method. The choice of local deformation energy parameter $\varepsilon$ is not to make a specific case study based on different grid, so reader can discuss the specific values under different circumstances further.

\section{ACKNOWLEDGEMENTS}

This work was supported by a grant from the Special for Scientific and Technological development of the Construction Cause of Qingdao (No. JK2014-34). 


\section{REFERENCES}

[1] Peng Qunsheng. Survey on Parameterization of Triangular Meshes[J]. Computer Aided Design \& Computer Graphics, 2004,16(6):732 737

[2] Zhong Li. Research of Triangular Mesh Parameterzation[D].2008(in Chinese)

[3] Yan Dongmei. Research of Triangular Mesh Technology[D].2005(in Chinese)

[4] Kent J R, Wayne E Carlson, Pichard E Parent. Shape transformation for polyhedral objects [A]. Computer Graphics Proceedings, Annual Conference Series, ACM SIGGRAPH, Chicago, 1992.47 54

[5] Alexa M. Recent advances in mesh morphing [J]. Computer Graphics Forum, 2002,21(2):173 196
[6] Kobbelt L P, Vorsatz J, Labisk U, et al. A shrink-wrapping approach to remeshing polygonal surfaces [J]. Computer Graphics Forum, 1999, 18(3): 119 129

[7] Shapiro A, Ayellet T. Polyhedron realization for shape transformation [J]. The Visual Computer, 1999, 14(8/9):429 444

[8] Hu Guofei, Fang Xiang, PengGunsheng. Convex Combination Spherical Parameterzation[J]. Computer Aided Design \& Computer Graphics, 2003,16(5):632 637(in Chinese)

[9] Quicken G, Brechbuhler M, Hug C. Parametrization of closed surfaces for parametric suface description [A]. Proceedings of IEEE Computer Society Conference on Computer Vision and Pattern Recognition, South Carolina, 2000. 354 360

[10] Xue Junxiao, Luo Zhongxuan. Energy Optimized Parameterization of Triangular Meshes[J]. Computer Aided Design \& Computer Graphics, 2009,21(10):1473 1482(in Chinese) 